\title{
Single atoms in the ring lattice for quantum information processing and quantum simulation
}

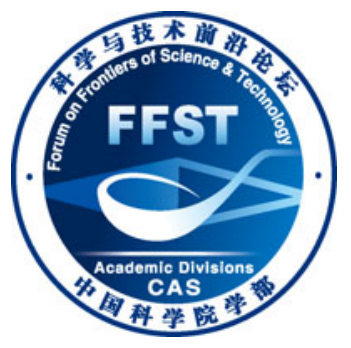

\author{
YU Shi ${ }^{1,2,3}$, HE XiaoDong ${ }^{1,2}$, XU Peng ${ }^{1,2}$, LIU Min ${ }^{1,2}$, WANG Jin ${ }^{1,2} \&$ ZHAN MingSheng ${ }^{1,2^{*}}$ \\ ${ }^{1}$ State Key Laboratory of Magnetic Resonance and Atomic and Molecular Physics, Wuhan Institute of Physics and Mathematics, Chinese \\ Academy of Sciences; Wuhan National Laboratory for Optoelectronics, Wuhan 430071, China; \\ ${ }^{2}$ Center for Cold Atom Physics, Chinese Academy of Sciences, Wuhan 430071, China; \\ ${ }^{3}$ Graduate University of the Chinese Academy of Sciences, Beijing 100049, China
}

Received February 13, 2012; accepted March 19, 2012

\begin{abstract}
We have demonstrated preparing and rotating single neutral rubidium atoms in an optical ring lattice generated by a spatial light modulator, inserting two atoms into a single microscopic optical potential efficiently by dynamically reshaping the optical dipole trap, trapping single atoms in a blue detuned optical bottle beam trap, and confining single atoms into the Lamb-Dicke regime by combining red and blue detuned optical potentials. In combination with the manipulation of internal states of single atoms, the study is opening a way for research in the field of quantum information processing and quantum simulation. In this paper we review the past works and discuss the prospects.
\end{abstract}

laser cooling and trapping, single atoms, quantum information processing, quantum simulation

Citation: $\quad$ Yu S, He X D, Xu P, et al. Single atoms in the ring lattice for quantum information processing and quantum simulation. Chin Sci Bull, 2012, 57: 19311945, doi: 10.1007/s11434-012-5153-8

Single neutral atoms are promising candidates for quantum information processing [1] and quantum simulation [2,3] besides trapped ions, photons, etc. The preparation and manipulation of single atoms in optical microscopic potentials open a route to deterministically controlling the quantum states. Due to the good scalability and long coherence time, these quantum systems have been employed to demonstrate quantum gate [4] and entanglement of atoms [5] based on "Rydberg blockade" effect [6,7]. A qubit can be encoded in the internal or motional states of an atom, and multi-qubit operations can be performed based on atom-light interactions or atom-atom interactions. In particular, the hyperfine ground states of alkali-metal atoms can be considered as qubits, which can be easily manipulated via microwave radiation or Raman transitions. Several kinds of proposals for quantum gate operation were designed based on dipoledipole interactions between Rydberg atoms [8], cavity-mediated photon exchange [9] and controlled collisions [10]. Fur-

*Corresponding author (email: mszhan@wipm.ac.cn) thermore, single atom array can serve as a research platform for physical models described in other systems that are not directly investigated experimentally. The few-body simulation offers an opportunity to understand some intriguing many-body phenomena, such as superconductivity in solid materials [11], and the natural process of photosynthesis [12].

Instead of being adiabatically transferred from ultracold atomic ensemble, in our experiment single atom array is built one by one based on "collisional blockade" mechanism $[13,14]$ that locks the atom number either zero or one in ultra small dipole trap in the presence of near-resonant laser light. Here, we demonstrate trapping single neutral rubidium atoms in the ring lattice generated by a computer controlled spatial light modulator (SLM), and present several kinds of manipulations of single atom array.

\section{Experimental overview}

Our experimental setup is shown in Figure 1 (see also [15]). 


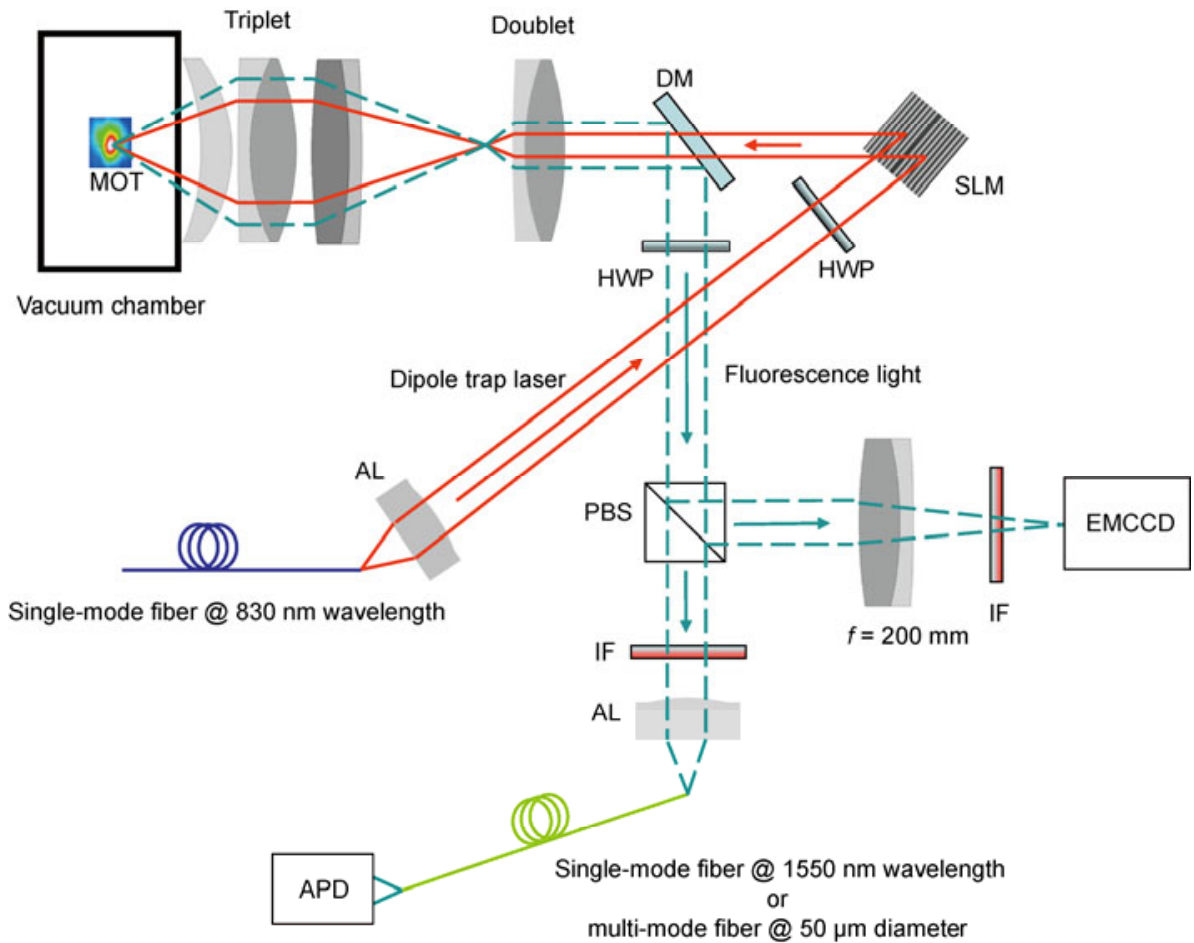

Figure 1 (Color online) Experimental setup for single atom trapping (solid line) and detection (dashed line). For simplicity, the magneto-optical trap (MOT) beams are not shown. SLM, spatial light modulator; DM, dichroic mirror; HWF, half-wave plate; PBS, polarization beam splitter; IF, interference filter; AL, aspheric lens; APD, avalanche photodiode; EMCCD, electron multiplying charge coupled device.

The SLM (Holoeye, HEO 1080P) has a resolution of 1920x 1080 pixels, and each pixel size is $8 \mu \mathrm{m} \times 8 \mu \mathrm{m}$. Controlled by computers, it could transform a single collimated Gaussian beam into arbitrary intensity pattern with first-order diffraction efficiency of $40 \%$. As we know, Laguerre-Gaussian (LG) beams possess orbital angular momentum along the optical axis with radial mode number $p$ and non-zero azimuthal mode number $l$ [16]. Among them, the $p=0$ modes, called doughnut beam, have a spiral phase structure where the phase is undefined on the optical axis [17]. The intensity distribution of these beams is given by

$$
I_{l}=I \frac{2}{\pi|l| !}\left(\frac{-2 r^{2}}{w(z)^{2}}\right)^{|l|} \exp \left(\frac{-2 r^{2}}{w(z)^{2}}\right),
$$

where $w(z)=w_{0} \sqrt{1+\left(z / z_{\mathrm{R}}\right)^{2}}, w_{0}$ is the beam waist, $z_{\mathrm{R}}$ is the Rayleigh length, and $I$ is the laser intensity. By interfering co-propagating two doughnut beams with opposite azimuthal indices $l$, a ring lattice shaped intensity distribution is obtained, which comprises $2 l$ petals [18]:

$$
I_{\text {ring }}=I \frac{2}{\pi|l| !}\left(\frac{-2 r^{2}}{w(z)^{2}}\right)^{|l|} \exp \left(\frac{-2 r^{2}}{w(z)^{2}}\right)(2+2 \cos (2 l \phi)) .
$$

Instead of superposing a pair of separated beams, the ring lattice in our experiment is generated by the SLM that imposes the holograms containing the phase of the superposed
LG modes onto a single laser beam. We implement an algorithm that has details in [19] using the MATLAB software to generate the ring lattice holograms. The function is

$$
\begin{aligned}
& \bmod \left[\left(\operatorname { a n g l e } \left[\mathrm{LG}\left(x, y, l, z, w_{0}, z_{\mathrm{R}}\right)\right.\right.\right. \\
& \left.\left.\left.+\mathrm{LG}\left(x, y,-l, z, w_{0}, z_{\mathrm{R}}\right)\right]+x \cdot k_{x}\right), 2 \pi\right] \cdot \frac{256}{2 \pi},
\end{aligned}
$$

where the term angle $\left[\mathrm{LG}\left(x, y, l, z, w_{0}, z_{\mathrm{R}}\right)+\mathrm{LG}(x, y,-l, z\right.$, $\left.\left.w_{0}, z_{\mathrm{R}}\right)\right]$ is the ring lattice phase pattern, and $x \cdot k_{x}$ is a blazed phase grating structure, acting as a tilted mirror to separate the ring lattice from the 0th-order non-modulated light. The phase pattern has one phase jump of $\pi$ for $l=1$ and $2 l$ symmetrical jumps of $\pi$ for $l>1$. The corresponding modes consist of $2 l$ petals (see Figure 2), with each petal as an optical dipole trap when the laser is red detuned from the atom resonance. One can notice that, for $l=0$ there is not phase jump, corresponding to the Gaussian mode which is a single trap with the same optical axis as the ring lattice.

Loaded from the ${ }^{87} \mathrm{Rb}$ magneto-optical trap (MOT) at the typical temperature of $100 \mu \mathrm{K}$, the generated ring lattice at $830 \mathrm{~nm}$ wavelength is strong focused to a waist of $2.1 \mu \mathrm{m}$ by a commercial microscope objective (NA $=0.38$, LINOS) placed outside the vacuum chamber. Because of aberration caused by the glass cell of the ultrahigh vacuum chamber, the focused system does not work in the diffraction-limited regime. The optical potential depth can be varied with the laser power, with a typical depth of $1 \mathrm{mK}$. The light induced fluorescence (LIF) of atoms in the ring lattice is collected 
(a)

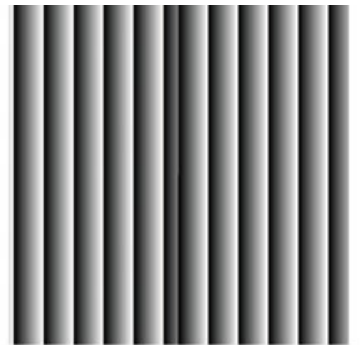

(b)

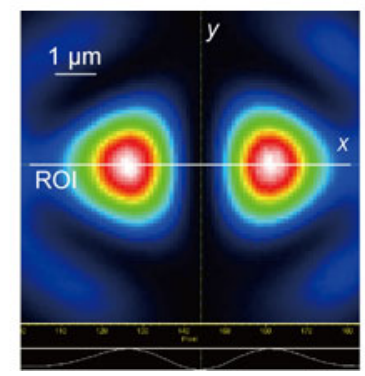

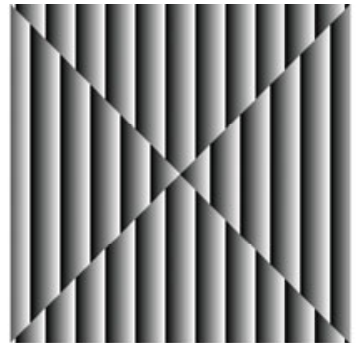

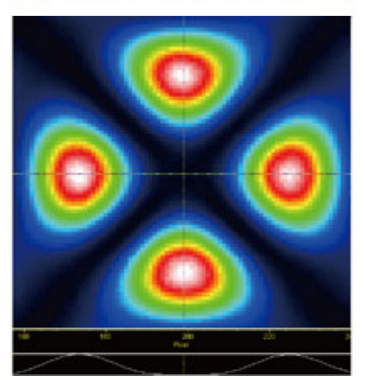

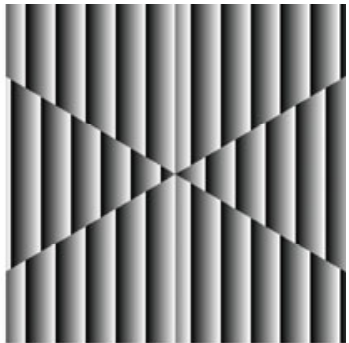

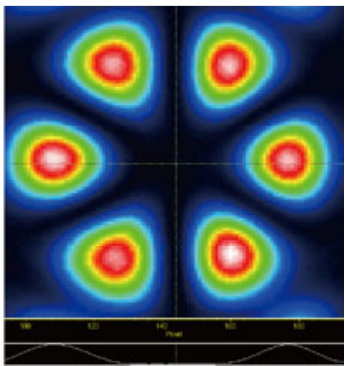

Figure 2 (Color online) Holograms calculated to generate the ring lattices of $l=[1,2,3]$ (a) and the corresponding optical intensity distribution observed by a CCD camera (b). The different gray levels in the holograms with $600 \times 600$ pixels represent different phase shifts from 0 to $2 \pi$.

with the same microscopic objective lens and separated from trapping beam with a dichroic mirror. Then it is divided into two parts. One is imaged onto an electron multiplying charge coupled device (EMCCD, PhotoMax 512) camera, and the other is coupled into a fiber and detected with an avalanche photodiode (APD) assembled in a single photon counting module (SPCM, EG\&G AQRH-14-FC). By selecting suitable fiber and adjusting the kinematical mounts of the collection system, we can collect the LIF from the whole lattice or any one trap. In the experiment, we choose a single-mode fiber at $1550 \mathrm{~nm}$ wavelength for spatial filtering or a multi-mode fiber at $50 \mu \mathrm{m}$ diameter for larger visual field.

By reducing the intensity and increasing the detuning of the MOT beams, the loading rate could be less than $1 \mathrm{~s}^{-1}$ for each microscopic far-off-resonance trap (FORT). Shown in Figure 3(a), we observe the fluorescence signals in an individual trap of the lattice in $20 \mathrm{~ms}$ time bins. The two characteristic steps represent no atom and one atom in the trap.

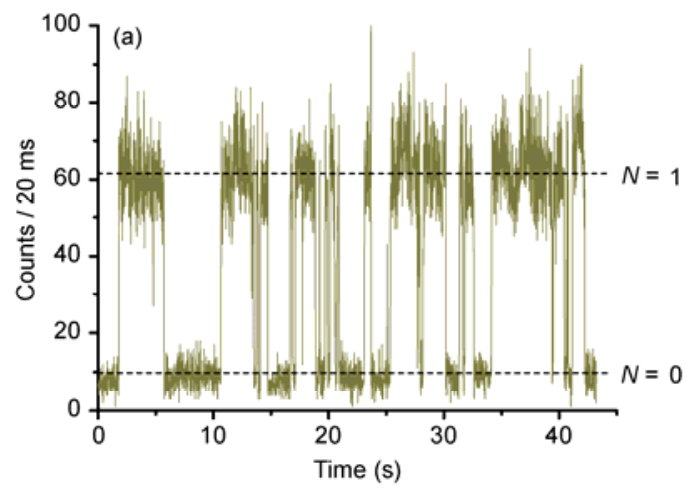

The sudden jumps of counts correspond to a single atom entering and being trapped, while the sudden drops result from atom leaving the trap due to a collision with another incident atom. The process of two or more atoms in a very small trap volume being ejected from the trap occurs as a result of light-assisted collision induced by the resonant laser at $780 \mathrm{~nm}$, known as the "collisional blockade" mechanism $[13,14]$. To assure that the upper fluorescence level corresponds to one atom, we make the Hanbury Brown and Twiss (HBT) effect measurement [20,21]. The fluorescence coupled into the single-mode fiber is sent through a 50/50 fiber optic beam splitter onto two APDs and then converted into NIM signal by discriminators (ORTEC 935). The delay time of photon pair events is recorded by a time-to-digital converter (TDC, RoentDek TDC8HP) only when the fluorescence exceeds the threshold in order to minimize background contributions. In Figure 3(b), the background corrected measurement results indicate that the fluorescence does come from one atom [21].

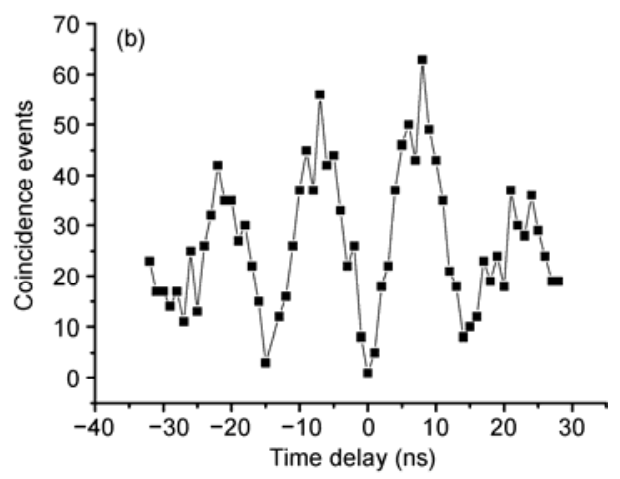

Figure 3 (Color online) Fluorescence signals of single atoms measured by an APD within 20 ms time bins [15]. (a) Number of photons counted in an individual trap. The steps correspond to either zero or one atom in the trap. (b) The background corrected coincidence events of single atom fluorescence measurement. When delay time $\tau$ is equal to zero, the corresponding coincidence event is zero. 


\subsection{The fluorescence signals and images of single atom array}

The LIF collection system is focused onto the whole lattice, so single atoms in the lattice could be monitored by the APD. The left picture of Figure 4 shows the fluorescence signals taken for two-trap and four-trap arrays with different coupled fibers. The signal to noise ratio with a multi-mode fiber (Figure 4(b)) is lower than one with a single-mode fiber (Figure 4(a)). It can be enhanced by improving the laser frequency stabilization and optimizing the laser intensity and frequency detuning [22]. The multiple jumps of counts indicate that atoms are trapped simultaneously in the ring lattice with one loaded in each trap. Since the loading process of single atoms is completely stochastic and independent, the probability of detecting more than one atom in the ring lattice is smaller than one atom in an individual trap [23].

Single atoms confined in the FORTs are separated by several micrometers. To observe atoms directly, we utilize an optical lens $(f=200 \mathrm{~mm})$ to focus the LIF onto the EMCCD camera with a magnification factor of about 20 to capture spatially resolved images. Appearing as each square in the images, one pixel of the camera is $16 \mu \mathrm{m} \times 16 \mu \mathrm{m}$, corresponding to a size of $0.8 \mu \mathrm{m} \times 0.8 \mu \mathrm{m}$ in the object plane. We overlap images of atoms with the camera photo surface and block background stray light with an interference filter. The fluorescent images of single atoms in the ring lattices are shown in the right picture of Figure 4 with an exposure time of $50 \mathrm{~ms}$.

\subsection{The lifetime of single atoms}

Single atoms cannot stay long in the dipole trap when the MOT is always on. We show a histogram of 770 single atom events, and fit it with an exponential decay function in Figure 5(a). The 1/e lifetime of single atoms with the MOT on is about $460 \mathrm{~ms}$, which is sufficient for us to judge and execute the time sequence in succession. For long-term storage, the MOT beams have to be switched off to stop loading process after an atom entering the trap, and only keeping one atom in the trap. The lifetime of single atoms in the FORT without the MOT beams is measured. In the experiment, we take the following time sequence: executing laser cooling and trapping process until the counts of fluorescence from an individual dipole trap exceed a threshold, then shutting off the MOT for a certain time while keeping the dipole laser on, after that switching on the MOT light again to detect whether the atom stay in the trap, then repeat. In Figure 5(b), we scan the off time from $2 \mathrm{~s}$ to $20 \mathrm{~s}$ to get the probabilities of atoms still in, and then fit the curve with an exponential decay function. Under our experimental (a)

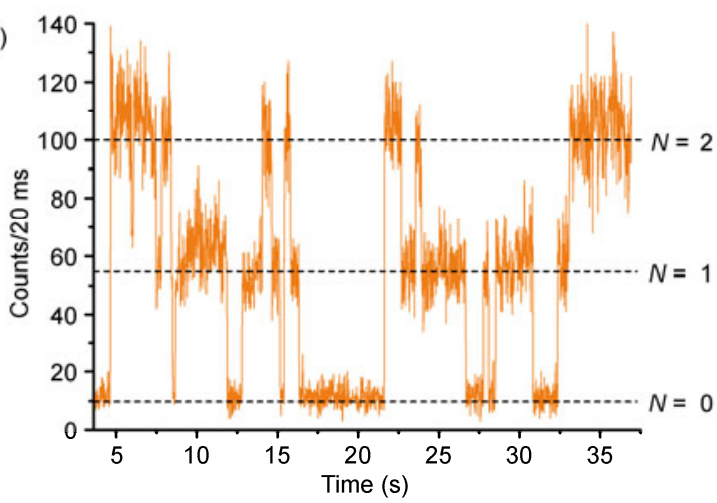

(b)

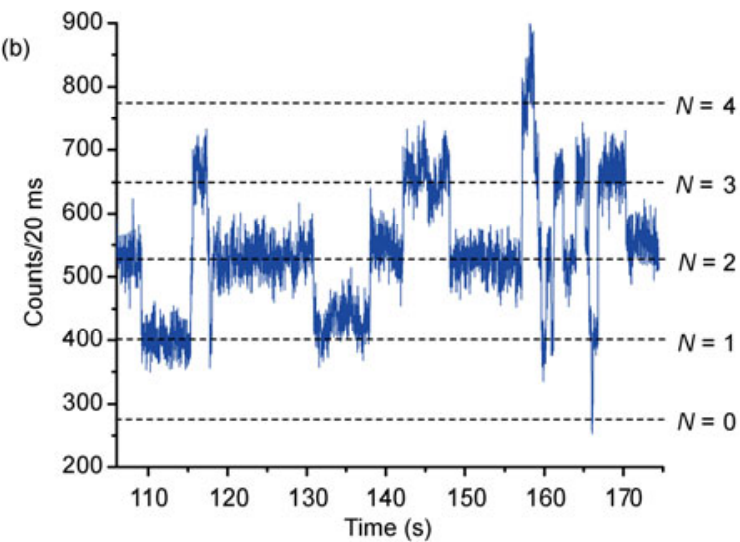

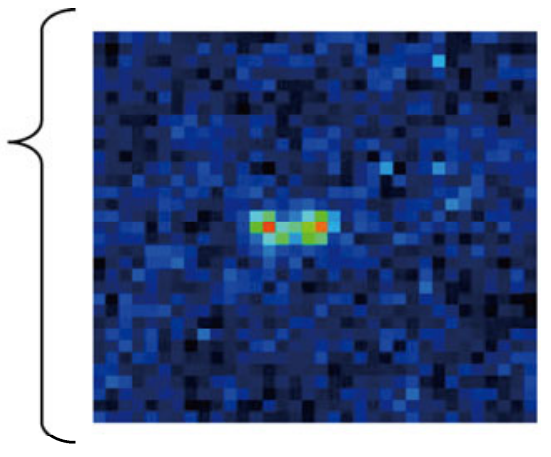

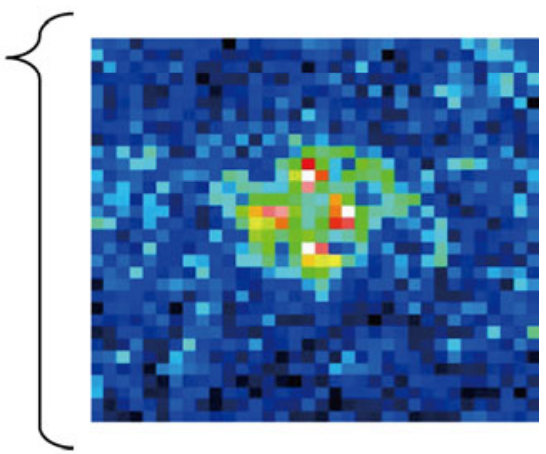

Figure 4 (Color online) Fluorescence signals of single atom array counted by an APD with different coupled fibers (left) and images captured by an EMCCD camera (right) for two-trap lattice of $l=1$ (a) and four-trap lattice of $l=2(\mathrm{~b})$. 

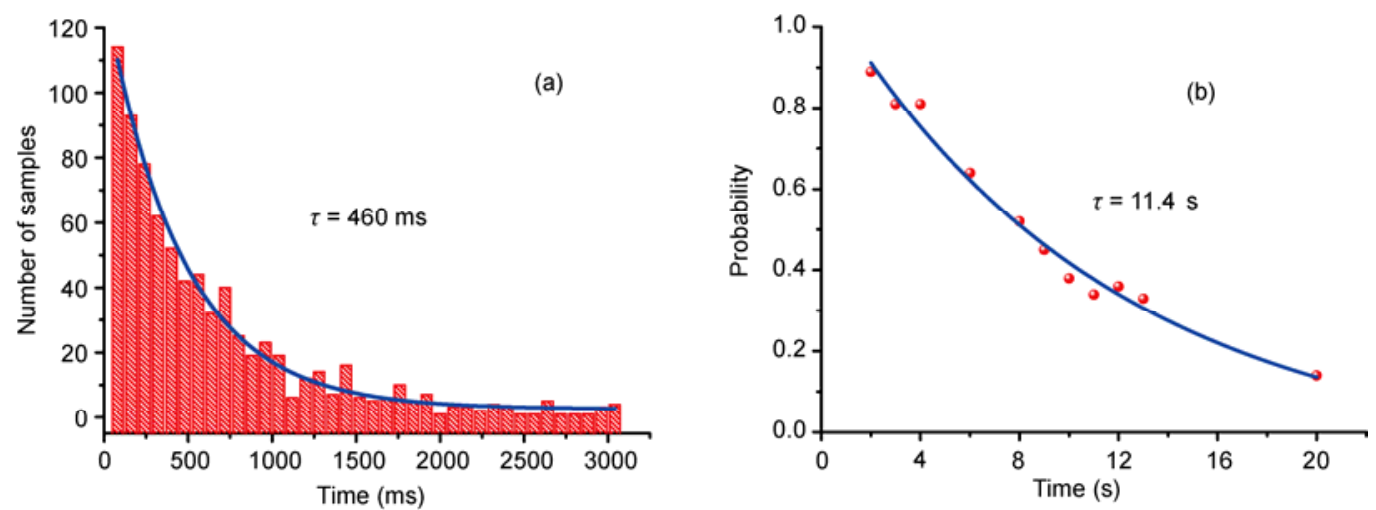

Figure 5 (Color online) Measurement of the lifetime with the MOT on and off. (a) Histogram of 770 single atom events measured by LIF with the MOT on. (b) Probability of single atoms still in the dipole trap without the MOT beams.

conditions, the $1 / e$ lifetime of single atoms is about $11.4 \mathrm{~s}$ due to collisions with untrapped background atoms in the ultrahigh vacuum chamber and heating mechanisms caused by intensity fluctuations and photon scattering of the FORT light [24-26].

\subsection{The oscillation frequencies and temperature of single atoms}

We expect to discuss the dynamic characteristic of trapped atoms by measuring oscillation frequencies and temperature in the FORT. It provides a basis for manipulations of atomic motion.

The optical potential $U(r, z)$ of atoms in the red detuned focused Gaussian beam can be written as

$$
U(r, z)=-U \exp \left(-\frac{2 r^{2}}{w(z)^{2}}\right)
$$

where $U$ is the maximum potential depth. If the mean kinetic energy of trapped atoms is much smaller than the potential depth, the optical potential can be approximated by a harmonic oscillator. In this case, the axial and radial oscillation frequencies of atoms are given by

$$
\begin{aligned}
& v_{z}=\frac{1}{2 \pi} \sqrt{\frac{2 U}{m z_{\mathrm{R}}^{2}}}, \\
& v_{r}=\frac{1}{2 \pi} \sqrt{\frac{4 U}{m w_{0}^{2}}} .
\end{aligned}
$$

For a trap depth of $U=1 \mathrm{mK}$, the calculated oscillation frequencies are about $5 \mathrm{kHz}$ and $50 \mathrm{kHz}$ in axial and radial directions respectively.

The oscillation frequencies of single atoms trapped in the FORT are measured by parametric excitation of the oscillatory motion. For a linear oscillator, parametric resonance occurs around the modulation frequencies $v_{\bmod }=2 v / n$, where $n$ is position integer. The width of resonance range and the amplification coefficient of oscillations rapidly de- crease with increasing $n$ [27]. In the experiment, once one atom in the FORT is detected, the MOT is turned off and the radio frequency power is sent to an amplifier that drives an electro-optic modulator (EOM) or acousto-optic modulator (AOM) to modulate the FORT potential depth. Then the MOT is switched on again to detect whether the atom is still in the trap. For a trap with depth $U=1 \mathrm{mK}$, we scan the modulation frequency from 3 to $22 \mathrm{kHz}$ with modulation amplitude of $10 \%$ in $200 \mathrm{~ms}$ and from 30 to $220 \mathrm{kHz}$ with amplitude of $8 \%$ in $100 \mathrm{~ms}$, shown in Figure 6(a) and (b). One can notice that, there are two dips with greatly different depth in each heating loss spectrum, corresponding to parametric resonances around $v_{\text {mod }}=v$ and $v_{\text {mod }}=2 v$. It indicates that the typical oscillation frequencies are $v_{z}=4.4 \mathrm{kHz}$ and $v_{r}=51 \mathrm{kHz}$, according with the theoretical values.

The temperature of single atoms is measured by the release and recapture $(\mathrm{R} \& \mathrm{R})$ method [28,29]. The energy distribution of single atoms loaded from the MOT and trapped in the FORT is thermal and follows the Boltzmann distribution. In order to extract temperature from the measurement, we compare the experimental data with calculation results obtained from the Monte Carlo simulations of the trajectories of single atoms. As shown in the Figure 6(c), the temperature of single atoms directly loaded from the MOT is $(41 \pm 1) \mu \mathrm{K}$ for a trap depth of $U=1 \mathrm{mK}$. The ratio between the FORT depth and the temperature is about 24. After taking the optimized laser cooling sequence, the sub-Doppler cooling process is implemented. The temperature of single atoms is lowered to $(13 \pm 1) \mu \mathrm{K}$ for a trap depth of $U=0.4$ $\mathrm{mK}$ with the ratio of 31 . Under this condition, the radial oscillation frequency is about $35 \mathrm{kHz}$, and the mean quantum number of the radial motion state is 7.2.

\section{Rotating single atoms in the ring lattice}

As described above, we have experimentally demonstrated trapping single neutral ${ }^{87} \mathrm{Rb}$ atoms in a ring lattice. Now we report the manipulation of the rotation of the lattice. For the first time, we two-dimensionally rotated single atom array 

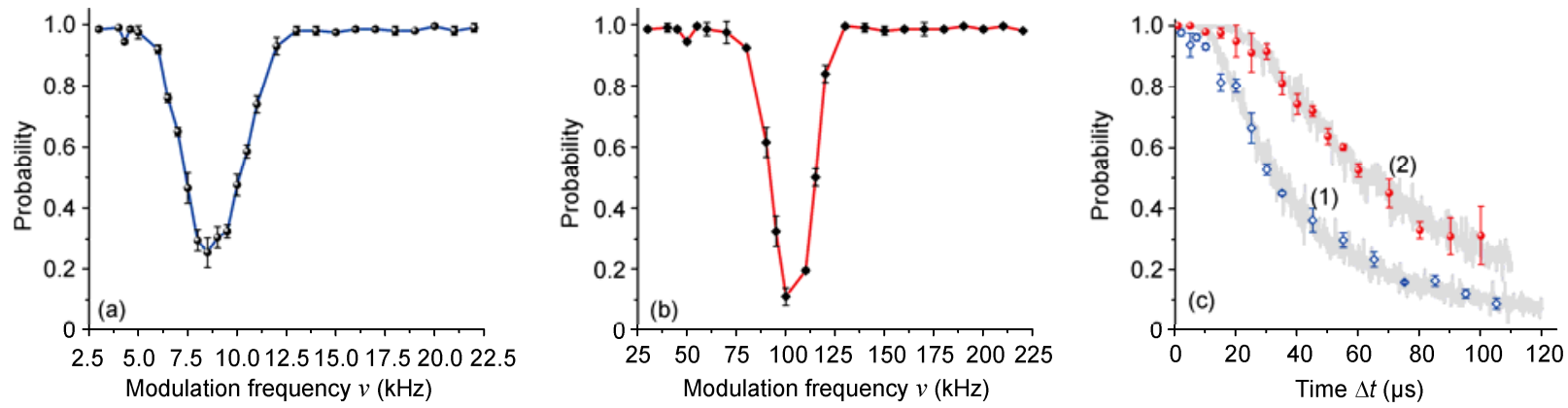

Figure 6 (Color online) Measurement of the axial and radial oscillation frequencies and temperature of single atoms in the FORT. (a) Probability of single atoms still in the trap after modulating the potential depth by $10 \%$ in $200 \mathrm{~ms}$. (b) Probability of single atoms still in the trap after modulating the potential depth by $8 \%$ in $100 \mathrm{~ms}$. (c) Probability of recapturing atoms as a function of release time. Each data point is the accumulation of 200 sequences. The gray line superimposed is a fit by the Monte Carlo simulation of the release and recapture method, which is the average of 200 trajectories for each release time. (1) The temperature of single atoms directly loaded from the MOT is $(41 \pm 1) \mu \mathrm{K}$ for a trap depth of $U=1 \mathrm{mK}$. (2) After optimizing the laser cooling sequence, the temperature is lowered to $(13 \pm 1) \mu \mathrm{K}$ for a trap depth of $U=0.4 \mathrm{mK}$.

by imposing a series of holograms onto the SLM with video resolution and refresh rates [15]. It can be observed from the modulated fluorescence signals of single atoms.

\subsection{Dynamically rotating the ring lattice}

In our experiment, the ring lattice laser has a phase pattern with $2 l$ symmetrical phase jumps of $\pi$ for $l>1$ and one phase jump of $\pi$ for $l=1$. The azimuthal position of intensity maxima can be varied with the positions of phase jumps. To generate rotating holograms, we can add an offset $\varphi$ to the azimuthal angle, i.e.

$$
\begin{aligned}
& \bmod \left[\left(\operatorname { a n g l e } \left[\mathrm{LG}\left(x, y, l, z, w_{0}, z_{\mathrm{R}}\right) \cdot \exp (i l(J-1) \varphi)\right.\right.\right. \\
& +\operatorname{angle}\left[\mathrm{LG}\left(x, y,-l, z, w_{0}, z_{\mathrm{R}}\right)\right] \cdot \exp (i l(J-1) \varphi) \\
& \left.\left.+x \cdot k_{x}\right), 2 \pi\right] \cdot \frac{256}{2 \pi} .
\end{aligned}
$$

To preserve the periodicity of the rotation progress and obtain maximum rotation rate, the offset azimuthal angle $\varphi$ is set to $2 \pi / N$, with $N$ satisfying $\bmod (60, N)==0$. The output holograms with a series of different values of $J$ (from 1 to 60 ), are converted to an audio video interleaved (AVI) version's video, which lasts for 1 second with maximum speed of 60 frames per second (fps). When playing the hologram movie on the desktop, the rotating holograms are displayed on the SLM with video resolution and the same refresh rates, thus the ring lattice rotates at a rate of $60 / \mathrm{N} \mathrm{Hz}$. From geometrical symmetry, values of $N=1,2$ do not change the shape of the ring lattice, corresponding to adding $2 \pi$ and $\pi$ step to the original azimuthal positions. Thus the maximum rotation rate obtained is $20 \mathrm{~Hz}(N=3)$ in this scheme.

Figure 7 shows the optical signals of the rotating $l=1$ ring lattice at different rotation rates of $6 \mathrm{~Hz}, 10 \mathrm{~Hz}$ and 15 $\mathrm{Hz}$ with the sample interval of $1 \mathrm{~ms}$. The signals are detected by a photo detector (New Focus, Model 1621) and recorded by a digital storage oscilloscope (Tektronix, TDS2014B). It suggests that the ring lattice is rotating smoothly in real time. The peak heights of signals are not the same, meaning that the laser intensities of two traps are not equal.

\subsection{Rotating the single atoms}

To monitor the rotating progress of single atoms in two-trap array, we first realign the fluorescence collection system and limit our observation view field only to one trap as in Figure 3(a). Once an atom is trapped, the MOT and repumping beams are switched off for $40 \mathrm{~ms}$ to induce the atoms trapped in the MOT diffusion out of the ring lattice region, and then the hologram video is played on the SLM with the atom being forced to rotate. The fluorescence signal is continuously recorded during the rotating process, which displays an oscillation with frequency equal to the
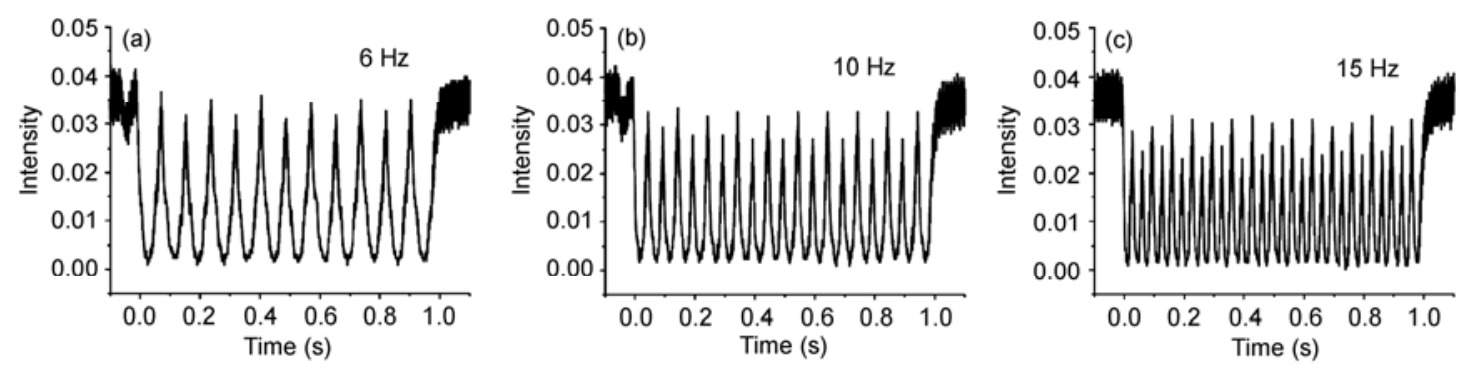

Figure 7 Optical signals of the rotating two-trap lattice for different rotation rates $60 / N \mathrm{~Hz}$ with $N=10$ (a), $N=6$ (b) and $N=4$ (c) [15]. The rotation lasts for $1 \mathrm{~s}$. 
rotation rate of holograms $(15 \mathrm{~Hz})$ due to the limitation of observation view field (see Figure 8(a)). Under this condition, if two atoms are trapped simultaneously in the ring lattice with one in each trap, the collection system would get twice maximum counting rate in one period. In Figure 8 (b), the fluorescence signal displays an oscillation with frequency $(12 \mathrm{~Hz})$ twice the rotation rate $(6 \mathrm{~Hz})$.

Another collection system is built to detect the LIF from north-pole position of the ring lattice where no trapped atom at the beginning (see the leftmost picture of Figure 2(b)). When single atom rotates and passes by the north-pole point, this collection system also sees the oscillating fluorescence signal with some time delay. In Figure 8(c), the fluorescence is recorded by aiming at the left trap (solid line) where the atom is initially trapped and the upper north-pole (dashed line). Since the ring lattice is clockwise rotating, the upper pole signal is about $1 / 4$ period later than the left trap signal. It indicates that the single atom is really rotating with the lattice.

Future research is planned to measure the decoherence time of rotating atoms and analyze the physical mechanisms leading to decoherence. We believe this scheme opens up possibilities for constructing quantum register with qubits encoded in the hyperfine levels of ground state of single atoms. The initial state preparation and single qubit operations can be achieved with Raman beams. The FORT laser can serve as one of the Raman pulses, as demonstrated in [30]; meanwhile the other is sent to the rotation region. Single qubit manipulation will be accomplished based on rotation. Furthermore, atomic qubits in the ring lattice could be simultaneously rotated by coupling the Raman beam into the same fiber as dipole trap laser. The ability of dynamic manipulation of single atoms also opens the route towards experiments to test schemes of atom-atom entanglement recommended in [23].

Technically, limited by the slow video refresh rate, the rotation rate of the ring lattice for single atoms is only 15 $\mathrm{Hz}$ at present. To overcome this limitation, we could utilize a versatile optical ring lattice and rotate the lattice smoothly and continuously in much faster speed [18]. However, for adiabatically transporting single atom in the trap, the acceleration $a$ of the rotating trap must fulfill $m a \sigma<<\hbar \Omega$, where $\Omega$ is the oscillation frequency of the atom, the mass is $m$, and the extension of the ground state wave function is $\sigma$ $[31,32]$. For our experimental parameters, $\mathrm{kHz}$ rotation rate of atoms could be obtained with this method.

\section{High efficient loading of two atoms into a microscopic optical trap}

In [33], a scheme for inserting two atoms initially stored in separate potential wells of a standing wave optical dipole trap into the same well is demonstrated, and then interaction between these atoms leading to light-induced collision is deterministically induced. However, the obtained success rate of preparing pairs of atoms is only $16 \%$ due to the uncertainty of distance control between the atoms. In our experiment, two single atoms initially trapped in double wells can follow the evolution of the computer controlled lattice and be transported into a microscopic FORT with higher efficiency. Under imposing the near resonance light, we observe the strong light-induce collisions between two atoms in a microscopic trap (see [34]).

\subsection{Dynamically reshaping the optical dipole trap}

The two-trap ring lattice and single Gaussian trap can be realized by the computer controlled SLM with the corresponding holograms calculated by eq. (3) for $l=1,0$. Instead of LG modes, a more precise description of the diffraction patterns produced by holograms with spiral phase structure and their superposition is provided by Kummer beams [35]. The amplitude of a monochromatic Gaussian beam after the Fraunhofer diffraction of holograms with $l$ index spiral structure could be described fully using the modified Bessel function of the first kind $I_{v}(z)$ [36]:

$$
u_{l}(r, \phi)=c_{0} i^{-l} \frac{\pi^{3 / 2} r^{3}}{32 w_{0}^{5}} \exp \left(i l \phi-\frac{r^{2}}{8 w_{0}^{2}}\right)\left[I_{(|l|-1) / 2}-I_{(|l|+1) / 2}\right] \text {. }
$$

The superposition of $u_{ \pm 1}$ from the eq. (8) is the amplitude of the double well. If $l=0$, amplitude distribution of the output beam is still Gaussian, corresponding to the single trap.
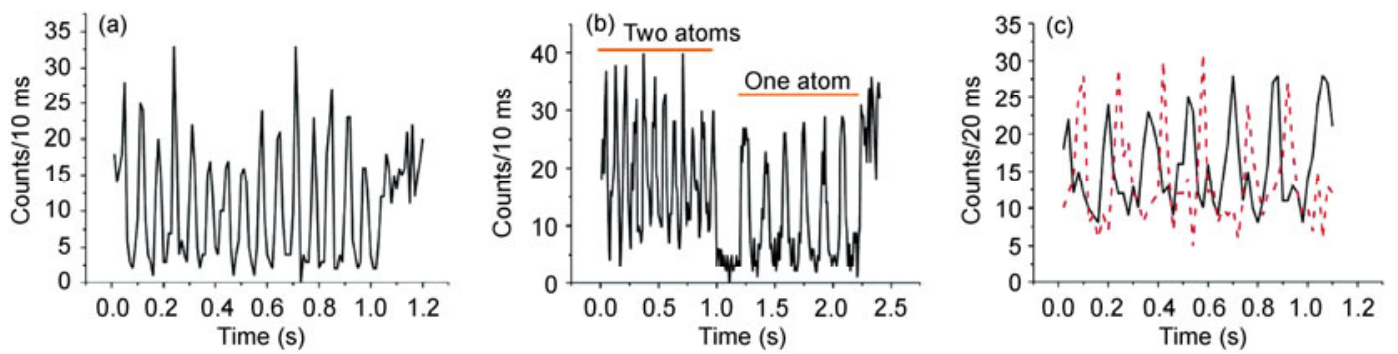

Figure 8 (Color online) Fluorescence signals of single atoms rotated in the two-trap lattice [15]. (a) Number of photons while one atom is trapped in the lattice. The observation view field is limited to an individual trap, and the rotation rate of the lattice is $15 \mathrm{~Hz}$. (b) Photon counts with one and two atoms in the lattice. The rotation rate is $6 \mathrm{~Hz}$. (c) Single atom signals recorded at the left trap (solid line) and the upper north-pole (dashed line). The rotation rate is 6 $\mathrm{Hz}$. 
Switching between the double well and single trap can be achieved by changing the holograms displayed on the SLM. This transformation process is the coherent sum of two electric field amplitudes of the diffracted Gaussian beam which can be described to be a linear mode:

$$
I(\xi)=\left|(1-\xi)\left[u_{1}(r, \phi)+u_{-1}(r, \phi)\right]+\sqrt{2} \xi u_{0}(r, \phi)\right|^{2},
$$

where $\xi$ is the percentage of Gaussian electric field amplitude component relative to the total electric field amplitude, which is time dependent linearly. The parameter $\xi$ evolves from 0 to 1 , corresponding to the transformation from double well to a single Gaussian trap. The cross-section evolution described by the eq. (9) is shown in Figure 9(a) and (b). The calculation result indicates that there is interference effect between two electric field amplitudes which will reduce the diffraction efficiency.

Experimentally, the corresponding output holograms are converted to an AVI version's video with $60 \mathrm{fps}$. The twotrap ring lattices could evolve into single traps in succession while playing the hologram movie on the desktop. The time evolution of trap transformation is monitored by the EMCCD camera. Due to the limitation of refresh rate, we choose to capture the image from the region of interest (ROI, see the leftmost picture of Figure 2(b)) in better time resolution, shown in Figure 9(c) and (d). The dip on the image data is in agreement with the theory that there is interference effect. The first-order diffraction efficiency decreases to $50 \%$ of the double well during the transformation process, resulting in escape of certain amount of trapped atoms.
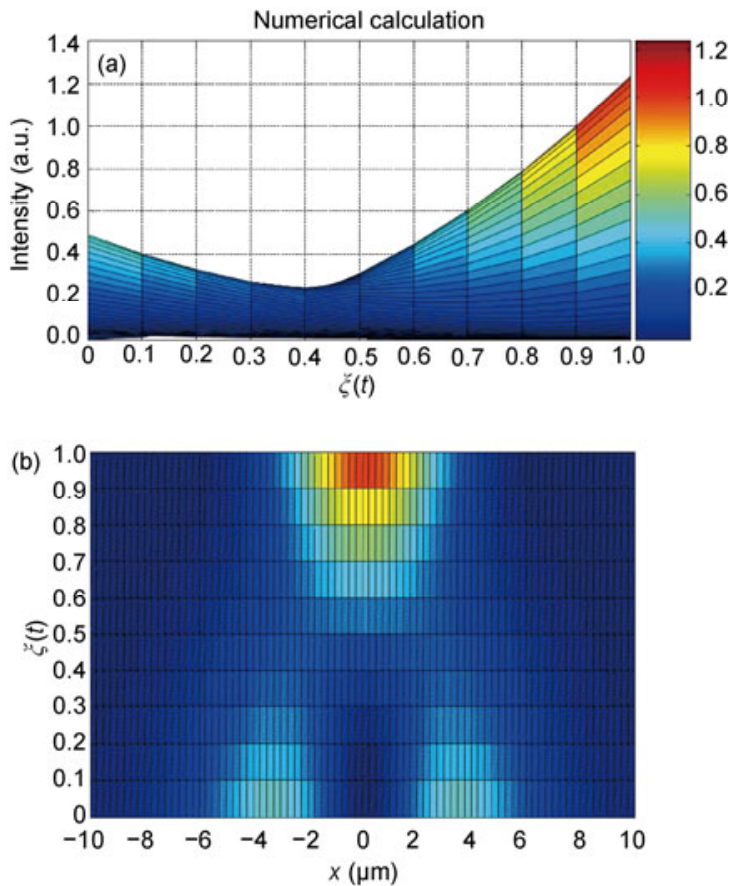

\subsection{Transporting two atoms into a single microscopic FORT}

In double well ring lattice, Figure 10(a) and (b) show the LIF from the whole two traps and single trap, respectively. Once the counting rate exceeds the blue line, meaning that two single atoms are trapped in the double well with one each, we trigger the control system to start the following experimental sequence: shutting off the MOT beams for 150 $\mathrm{ms}$ and playing the holograms movie to transform the trap shape and transfer atoms, then switching on the MOT beams to induce collisions and detecting atoms in the microscopic FORT for $60 \mathrm{~ms}$. The final result that two atoms could not stay in the same microscopic FORT with the MOT beams on is in accord with "collision blockade" [13] theory. The one body loss rate is about $15.5 \%$ derived from Figure 10(c).

In order to distinguish the atom loss induced by collisions from two uncorrelated atom loss during the transformation process, we carry out the entire experimental sequence with only one atom in double well to quantify the latter. As shown in Figure 10(d), one atom is transferred from lattice to a single trap with high success rate of $96.5 \%$. On the basis, we can infer that two atoms have been inserted into a single microscopic FORT at a success rate of $(96.5 \%)^{2}=93.1 \%$. While two atoms being loaded in the double well, the uncorrelated one-atom loss rate is $7.0 \%$ and the uncorrelated two-atom losses rate is about $0.1 \%$.

The one-atom loss during transformation process results from reduction of the first-order diffracting efficiency and
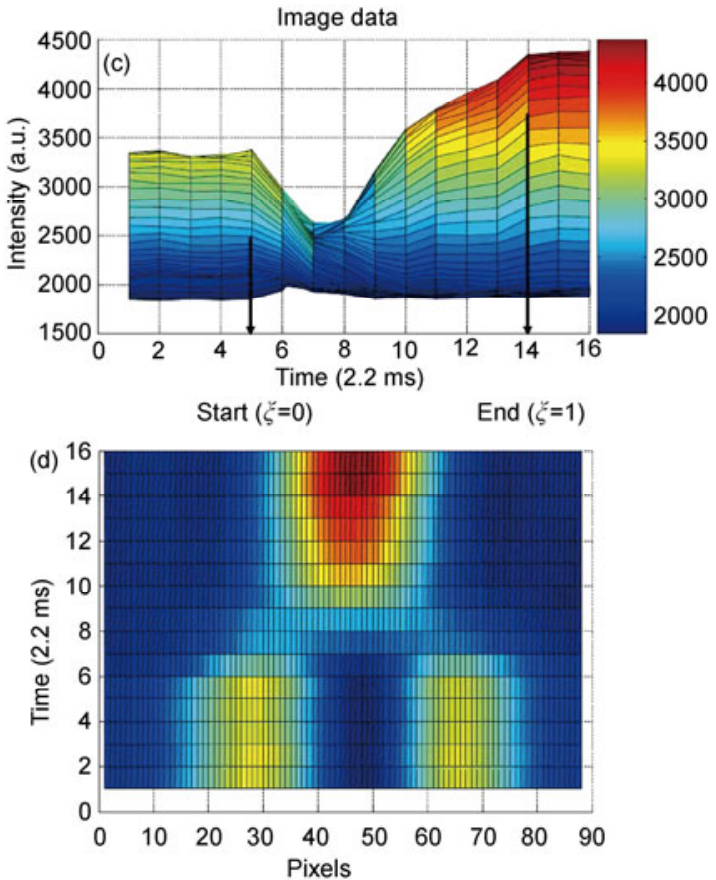

Figure 9 (Color online) Transformation between the double well and single Gaussian trap [34]. (a) and (b) are the numerical calculation of coherent transformation process, and go to $\xi$-intensity view and $\xi$-position view respectively. (c) and (d) are image data reconstruction captured from the region of interest, (c) goes to time-intensity view, and (d) is a pixel-time view. The arrows indicate the starting and ending time of the transformation process. 

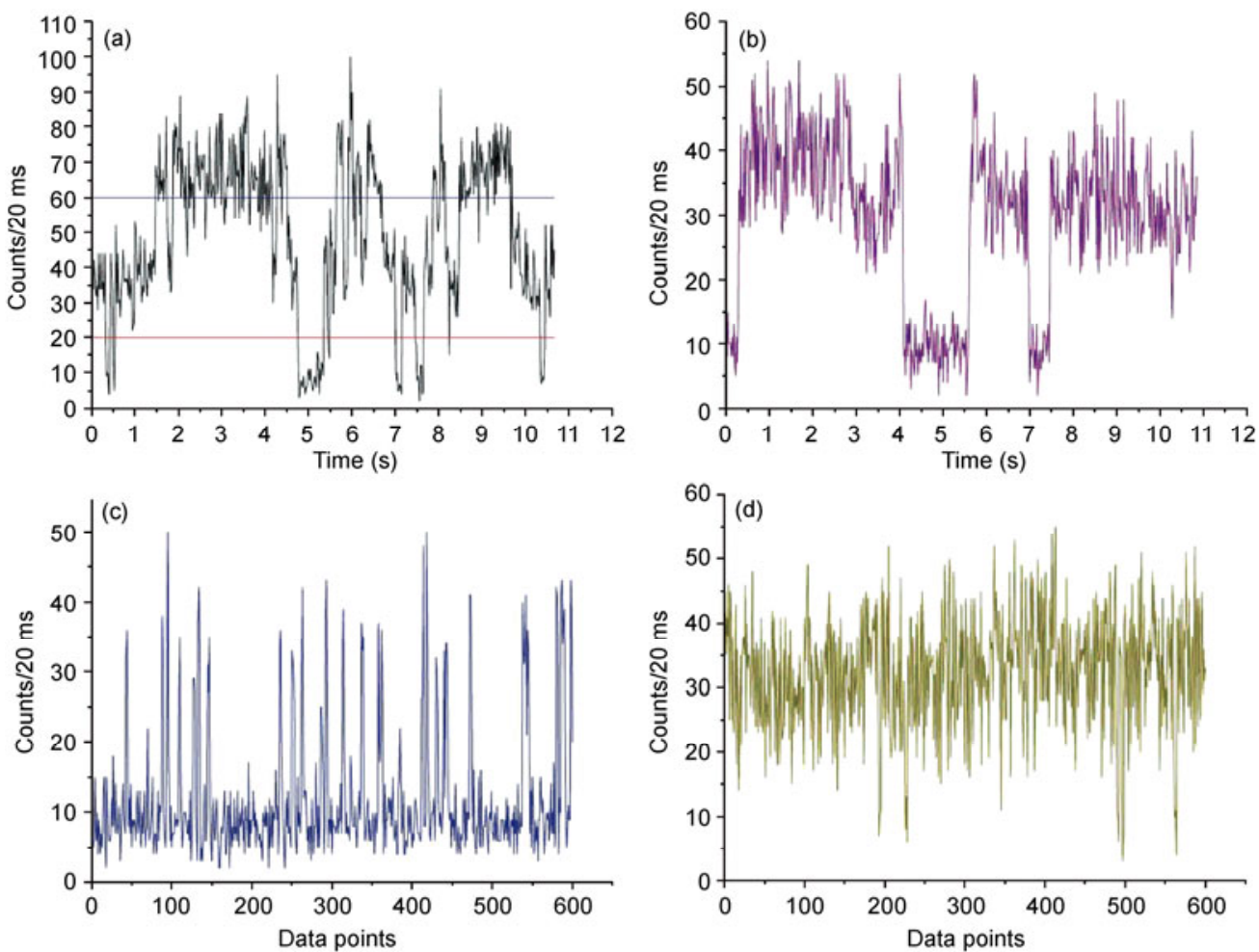

Figure 10 (Color online) Observed fluorescence signals of single atoms [34]. Each point corresponds to a $20 \mathrm{~ms}$ time bin. (a) Number of photons from the whole two-trap lattice. (b) Photon counts from one trap. (c) Accumulated fluorescence signals from single trap after two atoms trapped in the lattice being injected into the single trap. (d) Accumulated fluorescence signals from single trap after only one atom trapped in the ring lattice being inserted into the single trap.

trap potential, which would lead to atoms with higher energy escape. After the truncation of the Boltzmann distribution, the survival probability of atoms remaining in the trap is given by [28]

$$
P_{\text {surv }}(\beta)=1-\left(1+\beta+0.5 \beta^{2}\right) \exp (-\beta),
$$

where $\beta=E / k_{\mathrm{B}} T, E$ is trap potential, and $k_{\mathrm{B}} T$ is the mean energy of trapped atoms. Under our conditions, the survival probability of about $97 \%$ calculated from eq. (10) is in accordance with the measurement result. Therefore, the lower one-atom loss rate can be achieved by reducing the temperature of single atoms or raising the optical trap depth.

Collisions leading to atom loss in the presence of near-resonant laser light are governed by the long-range resonant dipole-dipole interaction including the radiative escape (RE) and fine-structure changing collisions (FCCs). They can be described by a simple semiclassical GallagherPritchard model [37,38]. Asymptotically, the potential is of the form $V_{S+P}=-C_{3} / R^{3}$ for one atom in the ground state and the other in the excited state. For the RE process, spontaneous emission of a photon red shifted from the atomic resonance can take place, and the resulting kinetic energy gained by collision atoms has a continuous distribution. If spontaneous emission does not occur, the atoms oscillate on the quasi-molecular potential curve until undergoing FCCs, which always causes an escape of both atoms from the shallow dipole trap due to the large fine structure splitting in rubidium atoms. Thus the RE collision process is the main reason for the correlated one-atom loss, with the probability of about $8 \%$ in our experiment.

With this simple and efficient method, we could also trap two single ${ }^{85} \mathrm{Rb}$ atoms and bring them together to show the isotopic difference in collisions [39] or combine one ${ }^{87} \mathrm{Rb}$ and one ${ }^{85} \mathrm{Rb}$ atom trapped to study the heteronuclear excited state-ground state collisions [40]. Utilizing two atoms determinately trapped in a microscopic FORT, we can discuss how van der Waals interactions lead to dephasing of the Rabi oscillations between ground and Rydberg states, and elucidate the role of Förster zero states in the dephasing [41]. Furthermore, if the motional state of atoms can be controlled arbitrarily, it opens the door for many fascinating experiments, e.g. producing single trapped diatomic molecules through Feshbach resonance [42] or photoassociation [43], preparing an entangled Bell pair of atoms based on coherent spin-changing collision between two atoms trapped in a single trap.

\section{Trapping single atoms in a blue detuned optical trap}

Instead of being trapped in the red detuned optical dipole trap (bright trap), several research groups have demonstrat- 
ed working with the blue detuned optical trap (dark trap) where the atoms are confined in a dark volume surrounded by laser beams $[17,44-47]$. In the dark trap, the photon scattering rate is greatly lessened, while in the bright trap it can be reduced only by increasing the detuning of the trapping laser. Meanwhile, the motional decoherence effect is significantly weakened due to elimination of the ac Stark shift in the optical dipole trap. The coherent time of atoms in a dark trap can thus be maintained to several seconds [48]. Another important application of the blue detuned trap may lay on equaling the polarizability for the ground and Rydberg state atoms [49]. We have demonstrated successfully trapping a single rubidium atom in a blue detuned optical bottle beam trap generated by the SLM [50]. Compared with other dark traps for single atoms $[45,46]$, our scheme has the advantage of both scalability and addressability, and the trapped atoms can be transported freely in space by well-controlled movement of the trap.

The optical bottle beam trap is generated by focusing a laser beam, whose phase is modified by the computer controlled SLM in place of the circular $\pi$ phase plate [44]. As shown in Figure 11(a), instead of circle, the central ellipse with $\pi$ phase shift in the hologram could compensate for the tilting angle between the incident laser beam and the SLM. Because of destructive interference between the central and outer parts of the laser beam, a dark region appears around the focus. The intensity distribution is calculated by numerically solving the Fresnel diffraction integral as [51] (see Figure 11(b))

$$
\begin{aligned}
E(\rho, \mu)= & \frac{1}{1-e^{-1}} \int_{0}^{1} r \exp \left(-r^{2}-0.5 i \mu r^{2}\right) J_{0}(\rho r) \mathrm{d} r \\
& -2 \frac{2}{1-e^{-1}} \int_{0}^{a} r \exp \left(-r^{2}-0.5 i \mu r^{2}\right) J_{0}(\rho r) \mathrm{d} r
\end{aligned}
$$

and

$$
I(\rho, \mu)=|E(\rho, \mu)|^{2},
$$

where $\rho=(N A) R \cdot 2 \pi / \lambda$ and $\mu=(N A)^{2} Z \cdot 2 \pi / \lambda$ with the radial coordinate of $R$ and the axial coordinate of $Z, a$ is the normalized radius of the phase shift, and the wavelength of the dipole trap laser is $\lambda$.

In the experiment, the blue detuned optical dipole laser at the wavelength of $772 \mathrm{~nm}$ from a tunable Ti:sapphire laser (Coherent MBR-110) is transformed by the SLM and strongly focused by the microscope objective above. In Figure 11(c), the intensity cross sections near the focus recorded by CCD are in agreement with the theoretical calculation results. While a little difference in diameter is due to the imperfection in focusing and propagating process. Obviously, it reveals that the radius of surrounded dark region in the focal plane is about $3 \mu \mathrm{m}$. The intensity ratio between the trap center and the wall, also called darkness, is meas-

ured to be $1: 200$ by scanning through the focal plane with a pinhole. According to [52], the optical dipole laser power of $80 \mathrm{~mW}$ used in our experiment corresponds to the dark trap depth of about $1 \mathrm{mK}$. Different from collection system in bright trap shown in Figure 1, a polarization beam splitter separates the LIF from the optical dipole laser while losing half of the signals. Through two interference filters (Semrock LL01-780-12.5), the fluorescence is coupled into fiber and guided to the APD.

In order to load atoms from the MOT to the dark trap, the dipole laser is shut off for a while and then switched on again to hold atoms (the capturing cycle). The capturing cycle is performed continuously until the counts of fluorescence exceed the threshold, meaning that a single atom has been captured, then the laser is kept on (the holding cycle) until the counts fall below the threshold. In Figure 12(a), the sudden raising and falling of the counts is similar to that in bright trap, meaning that an atom enters and leaves the trap. Figure 12(b) shows a histogram of the counts recorded in a total time of $2600 \mathrm{~s}$ and a compound Poisson law fitted to the 0 -atom and 1 -atom peaks. It indicates that we have actually compressed the trapping volume to the "collisional blockade" regime, in which more than one atom could not

(a)

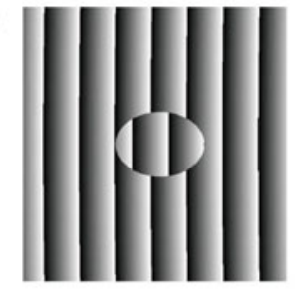

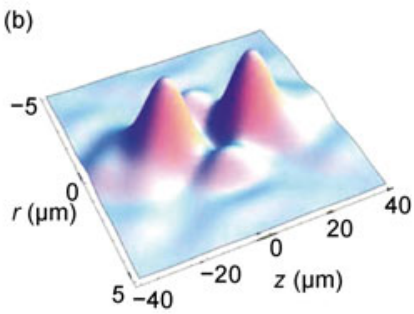

(c)

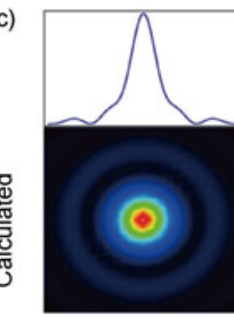

$z_{R} / 2$

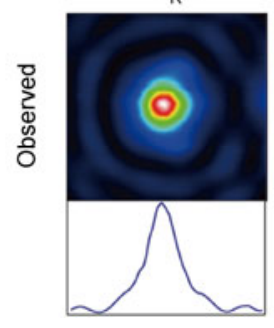

Figure 11 (Color online) Generation for the blue detuned optical bottle beam trap [50]. (a) Calculated hologram displayed on the SLM for the dark trap. (b) Calculated intensity distribution with $a=0.62, \mathrm{NA}=0.29$, and $\lambda=$ $772 \mathrm{~nm}$. (c) Intensity cross sections and corresponding beam profiles near the beam focus. Images are theoretically calculated (top) and experimentally observed (bottom), respectively, along the optical axis away from the focus with $0, z_{\mathrm{R}} / 4$, and $z_{\mathrm{R}} / 2$, where $z_{\mathrm{R}}$ is the Rayleigh range. 

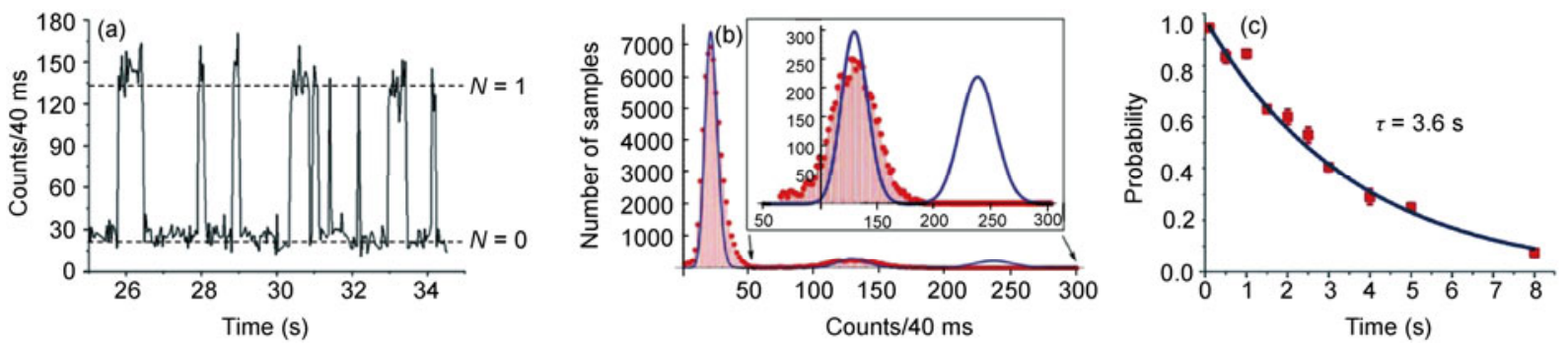

Figure 12 (Color online) Fluorescence signals and lifetime measurement of single atoms in the dark trap [50]. (a) Number of photons counted by an APD with $40 \mathrm{~ms}$ time bins. (b) Histogram of photon counting data lasting for $2600 \mathrm{~s}$ (dot) and the fit with a compound Poisson law for the zero-atom, one-atom peaks (solid curve). The two-atom peak is clearly missing. (c) Measurement of the lifetime with the MOT off. Each point is averaged three times, and each time includes 100 events.

be stably trapped. In Figure 12(c), we scan the off time from 0.1 to $8 \mathrm{~s}$ and fit with an exponential decay function to measure the lifetime of single atoms in the dark trap without the MOT beams. The $1 / e$ lifetime is $3.6 \mathrm{~s}$, which is long enough for the next step of the experimental requirement. By replacing the SLM with a $\pi$ phase plate to reduce the intensity noise in the present experiment, and by more carefully adjusting the trap parameters to lower the darkness of the dipole trap, the lifetime of single atoms in the blue detuned dipole trap can be much longer.

Recently, trapping and quantum state control of single Cesium atoms in a $532 \mathrm{~nm}$ wavelength bottle beam trap formed by crossing two unit charge vortex beams are demonstrated in [53]. The fast Rabi oscillation with a coherence time of about $43 \mathrm{~ms}$ is performed. In the future, we hope to achieve the long coherence time in the optimized dark trap which is anticipated to be at least several seconds [6]. We believe it will have more applications in quantum information processing and quantum simulation based on laser trapped atoms.

\section{Combining red and blue detuned optical potentials to form a Lamb-Dicke trap}

In order to precisely manipulate the internal and external degrees of freedom simultaneously, we expect to further cool single atoms to the ground vibrational state by implementing Raman sideband cooling [54,55] or electromagnetically induced transparency (EIT) cooling [56]. Similar to single trapped ions [54,57], single atoms need to be confined into the Lamb-Dicke regime where transitions that change the motional states are strongly suppressed. The quantitative description is given by

$$
\eta^{2}(2 n+1)<<1,
$$

where the Lamb-Dicke parameter $\eta=\sqrt{E_{r} / \hbar v}$, and $E_{r}$ is the recoil energy of atoms, $v$ is the oscillation frequency of single atoms and $n$ is the motional quantum number of the atomic harmonic oscillator state. To construct a LambDicke trap, we need to increase the oscillation frequencies.
The axial oscillation frequency in our experiment is much small due to extremely weak axial confinement of single atoms, which can be greatly increased in a standing wave field [21]. To enhance the radial confinement, it is a good approach to reduce the waist of the focused spot. However, limited by the technology, it is difficult to make the focus small enough. At present, by using diffraction-limited optics based on the combination of a large numerical aperture aspheric lens $(\mathrm{NA}=0.5)$ placed inside the vacuum chamber and a few standard lenses placed outside, a waist of $w_{0}=$ $(1.03 \pm 0.01) \mu \mathrm{m}$ at $850 \mathrm{~nm}$ and a radial oscillation frequency of $v_{r}=(160 \pm 3) \mathrm{kHz}$ with a trap depth $U_{0}=2.8 \mathrm{mK}$ have been obtained $[28,58]$. If stronger confinement is required and at the same time a lower red detuned potential depth is desired, a different method must be used. In [59], we have successfully demonstrated a new scheme to enhance efficiently the radial oscillation frequency and confine single atoms into the Lamb-Dicke regime by a non diffractionlimited optical system.

\subsection{Theoretical model for bichromatic far-off- resonance trap}

The key to our scheme is the use of a bichromatic far-offresonance trap (BFORT) which is composed of a blue detuned LG beam and a red detuned Gaussian beam. Since the atoms are attracted to the intensity maximum for a red detuned dipole potential and pushed to the intensity minimum for a blue detuned dipole potential, it is possible to combine the red detuned attractive dipole force and blue detuned repulsive dipole force to form a stronger force for the atoms.

Experimentally, we could overlap a blue detuned doughnut $\left(\mathrm{LG}_{p=0}\right)$ beam with a red detuned Gaussian beam to greatly squeeze the radial trapping dimension of single atoms. The radial position of the maximum intensity is related to $l$ by $r_{\max } \propto \sqrt{l / 2}$. This indicates that a doughnut beam with $l=1$ will have the smallest dark spot. At the focus, the optical dipole potential $U_{b}(r)$ of ${ }^{87} \mathrm{Rb}$ atoms for a blue detuned LG beam $(p=0, l=1)$ can be written in terms of the maximum potential depth $U_{b \max }$ as 


$$
U_{b}(r)=e U_{b \max } \frac{2 r^{2}}{w_{10}^{2}} \exp \left(-\frac{2 r^{2}}{w_{10}^{2}}\right)
$$

where $w_{10}$ is the waist size of the blue detuned laser beam, and $e$ is the Euler's number $e \approx 2.718$. According to eq. (4), the optical potential $U_{r}(r)$ for a red detuned Gaussian beam at the focus has the form in terms of maximum potential depth $U$ :

$$
U_{r}(r)=-U \exp \left(-\frac{2 r^{2}}{w_{0}^{2}}\right)
$$

The total optical dipole potential is given by the superposition of these two optical dipole potentials with opposite signs:

$$
\begin{aligned}
U_{\text {total }}(r) & =U_{b}(r)+U_{r}(r) \\
& =e U_{b \max } \frac{2 r^{2}}{w_{10}^{2}} \exp \left(-\frac{2 r^{2}}{w_{10}^{2}}\right)-U \exp \left(-\frac{2 r^{2}}{w_{0}^{2}}\right) .
\end{aligned}
$$

Figure 13 shows the cross-section of potentials described by eqs. (14)-(16) with $U_{b \max }=U$ and $w_{10}=w_{0}$. It indicates that the synthetic potential is steeper than Gaussian dipole trap. In harmonic approximation, the optical potential eq. (16) can be given by

$$
U_{\text {total }}(r) \approx-U+\frac{2\left(e U_{b \max } w_{0}^{2} / w_{10}^{2}+U\right) r^{2}}{w_{0}^{2}} .
$$

Calculated from eq. (17), the radial oscillation frequency $v_{\text {eff }}$ of single atoms trapped in the BFORT can be expressed simply in terms of the gain factor $\mathrm{g}$ and the radial oscillation frequency $v_{\mathrm{r}}$ in the Gaussian FORT:

$$
v_{\text {eff }}=g v_{r}=\left(\frac{e U_{b \max }}{U} \frac{w_{0}^{2}}{w_{10}^{2}}+1\right)^{1 / 2}\left(\frac{4 U}{m w_{0}^{2}}\right)^{1 / 2} \frac{1}{2 \pi} .
$$

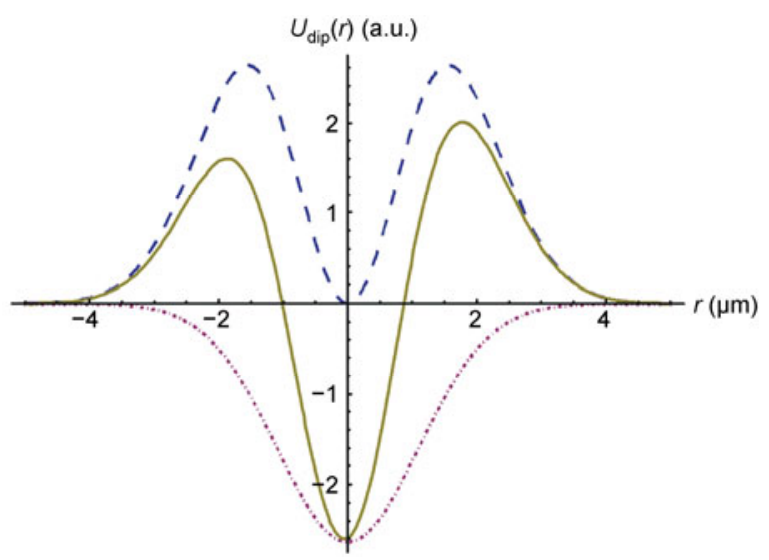

Figure 13 (Color online) Calculated cross-section of optical dipole potentials for blue detuned doughnut beam (dashed), red detuned Gaussian beam (dot-dashed) and BFORT (solid line). The blue detuned beam and red detuned beam have the same waist and potential depth.
Therefore, for $U_{b \max }=U$ and $w_{0}=w_{10}$, we can achieve a trap steeper than the Gaussian trap and enhance the oscillation frequency by $\sqrt{e+1} \approx 1.9$, as shown in Figure 13. For $U_{b \max }=10 U$ and $w_{0}=w_{10}$, the gain factor is $\sqrt{10 e+1} \approx 5$.3. If the original waist of the focused Gaussian spot is $1 \mu \mathrm{m}$, the effective trapping waist for single atoms can be amazingly reduced to $0.2 \mu \mathrm{m}$. This value is much smaller than the diffraction-limited spots $1.22 \lambda / \mathrm{NA}$ of the objective with maximum numerical aperture $\mathrm{NA}=1$ and focused dipole laser wavelength $\lambda=830 \mathrm{~nm}$.

\subsection{Experimental demonstration for the Lamb-Dicke trap}

In order to generate the doughnut beam, a blue detuned Gaussian laser beam $(\lambda=770 \mathrm{~nm})$ from the Ti:Sapphire laser is reflected by the SLM with a "fork" type phase hologram. The SLM phase modulation creates a "charge-one" phase singularity in the beam, centered on the "fork" defect. The collimated blue detuned doughnut beam is combined with the red detuned Gaussian beam $(\lambda=830 \mathrm{~nm})$ by a polarization beam splitter, and then focused simultaneously onto the MOT region. The fluorescence is separated from the blue detuned and red detuned beams by a dichroic mirror, then coupled into the single mode fiber and detected by the APD.

The optical intensity distributions observed by the CCD camera are shown in Figure 14(a) and (b). Extracted from line profile fitting in Figure 14(c) and (d), the waists of the blue and red detuned beams are about $w_{10} \approx 2.30 \mu \mathrm{m}$ and $w_{0} \approx 2.1 \mu \mathrm{m}$, respectively. Because of the repulsive potential for atoms from the blue detuned doughnut beam, single atoms are loaded into the red detuned dipole trap with potential depth of $U=1 \mathrm{mK}$ from the MOT while the doughnut beam is off. Once one atom is trapped, the doughnut beam is adiabatically ramped up over $10 \mathrm{~ms}$. The potential depth of doughnut beam is about $U_{b \max }=3.9 \mathrm{mK}$. Calculated from eq. (18), the oscillation frequency of single atoms could be enhanced by a factor of 3 under this condition.

The oscillation frequencies of single atoms trapped in the BFORT are measured by modulating the Gaussian beam potential depth. Similar to the measurement spectrum in Figure 6(a), the typical axial oscillation frequency in the BFORT is the same as one in the corresponding FORT. For the radial oscillation frequency measurement, we find that the intensity of resonance around $150 \mathrm{kHz}$ is much stronger than resonance around $300 \mathrm{kHz}$ under the same modulation conditions. To obtain the clear heating loss spectrum, we scan the modulation frequency from 40 to $190 \mathrm{kHz}$ with $1.9 \%$ amplitude modulation in $100 \mathrm{~ms}$ and from 200 to 450 $\mathrm{kHz}$ with increased $12 \%$ amplitude modulation in $100 \mathrm{~ms}$, shown in Figure 15(a). The experimental results around 150 $\mathrm{kHz}$ do not match the characteristics of parametric resonance. Because of experimental imperfections, there is a 

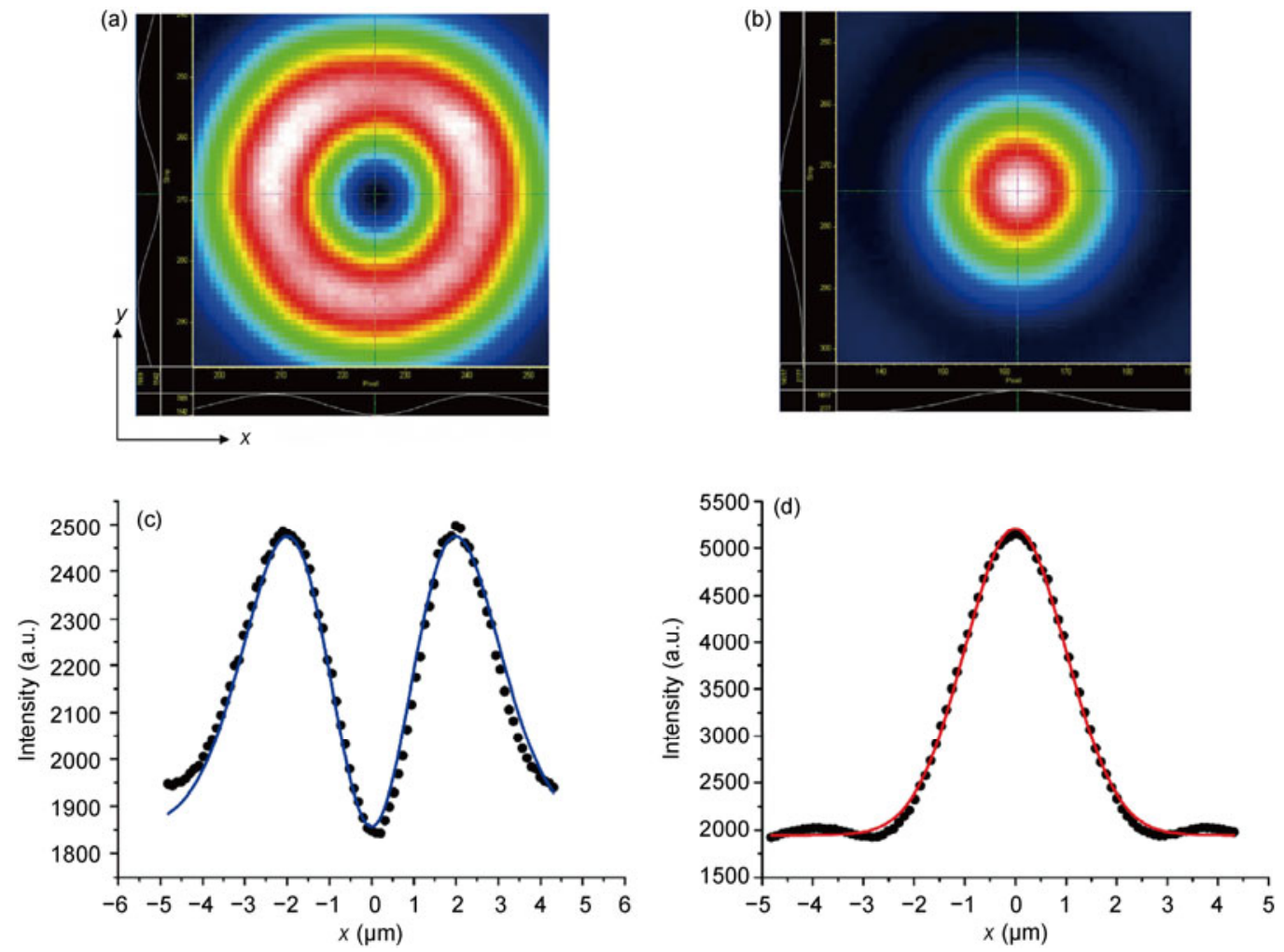

Figure 14 (Color online) Images ((a) and (b)) and line profile fittings ((c) and (d)) of focused doughnut spot and symmetric Gaussian spot [59].
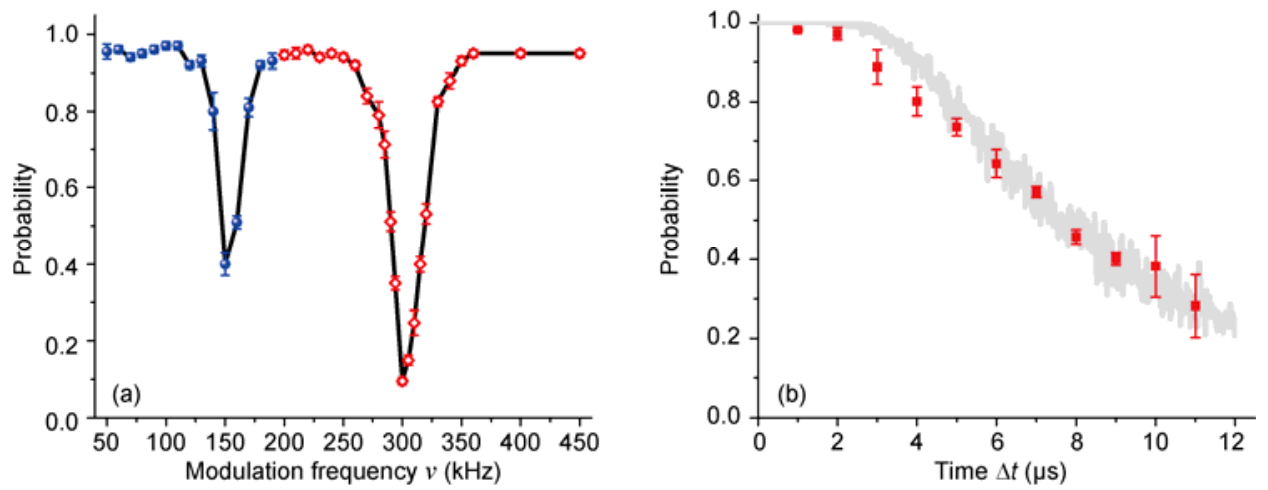

Figure 15 (Color online) Measurement of the radial oscillation frequency and temperature of single atoms in the BFORT. (a) Probability of single atoms still in the trap after modulation of the Gaussian beam potential depth as a function of the modulation frequency. (b) Probability of recapturing atoms as a function of release time after the sub-Doppler cooling process. Each data point is the accumulation of 200 sequences. The gray line superimposed is a fit by the Monte Carlo simulation of the release and recapture method, which is the average of 200 trajectories for each release time. The temperature is about $(15 \pm 1) \mu \mathrm{K}$

certain displacement between the optical axis of the Gaussian and doughnut beams. The equilibrium position of single atoms trapped in the BFORT may depend on the ratio of the attractive potential to the repulsive one. If the Gaussian beam is modulated, the equilibrium position will shake accordingly. When the modulation frequency is equal to the intrinsic trap frequency of single atoms, shaking will increase the oscillation amplitude and lead to heating [26,27]. It indicates that the radial oscillation frequency of single atoms in the BFORT is increased to $150 \mathrm{kHz}$, which agrees reasonably well with the theoretical expectation. As de- scribed by eq. (18), the frequency enhancement factor depends on the ratio of the blue detuned potential depth to red detuned potential depth. It allows us to adjust the radial oscillation frequency continuously by changing the blue detuned potential depth. Furthermore, we can optimize our scheme by using a diffraction-limited optics as in [58].

After the sub-Doppler cooling process, the temperature of single atoms trapped in the BFORT is $(15 \pm 1) \mu \mathrm{K}$, as shown in Figure 15(b). Under this condition, the mean quantum number of the radial oscillator is about 1.65 , and the Lamb-Dicke parameter is about 0.16 . This gives the rela- 
tion $\eta^{2}(2 n+1) \approx 0.11$ and indicates that eventually single atoms is confined into the Lamb-Dicke regime. It is a good starting point for implementing Raman sideband cooling [55] or sideband cooling by driving microwave transitions [60] to further cool single atoms down to the ground state.

\section{Conclusions and outlook}

In summary, we have trapped single rubidium atoms in a red detuned ring lattice generated by the SLM. The trapping time is several seconds, and temperature is about $13 \mu \mathrm{K}$ via sub-Doppler cooling. We have also experimentally demonstrated rotating single atom array, which opens up possibilities for single qubits manipulation and addressing. In order to investigate interactions between two atoms in a single trap, we have inserted two individual atoms into a single microscopic FORT with high efficiency by dynamically reshaping the trap. Single atoms have also been trapped in a blue detuned optical bottle beam trap. It will have more applications in quantum information processing because of reduced photon scattering and motional decoherence effects. Moreover, combining red and blue detuned optical potentials, the radial oscillation frequency has been enhanced efficiently, and single atoms have been confined into the Lamb-Dicke regime after the sub-Doppler cooling process.

For future research, we will manipulate the hyperfine states and excite Rydberg states of single atoms by a twophoton transition. Rydberg atoms exhibit bizarre properties, such as strong dipole-dipole interactions and sensitivity to external electric field [61]. Our single atom array is an ideal platform for forming Rydberg rings, which have been analyzed theoretically recently in references [62-64]. Due to "Rydberg blockade" effect [6,7], this system is ideally suited to perform multi-qubit operations, investigate quantum phenomena in strong interactions and experimentally simulate the energy transport procedure in light-harvesting complexes [12]. Furthermore, based on the construction of the Lamb-Dicke trap, it is possible to further cool single atoms down to the motional ground state. Thus we could precisely control the internal and external degrees of freedom simultaneously. It will provide a chance to carry out many fascinating experiments, such as efficiently producing single cold diatomic molecules using Feshbach resonance [42], preparing an entangled Bell pair of atoms by exploiting coherent spin-changing collisions between two atoms trapped in a single trap. All of these works will finally lead to the quantum information processing and quantum simulation with neutral atoms.

This work was supported by the National Basic Research Program of China (2012CB922101), the National Natural Science Foundation of China $(11104320,11104321$ and 10804124) and the Chinese Academy of Sciences.

1 Nielsen M A, Chuang I L. Quantum Computation and Quantum Information. Cambridge: Cambridge University Press, 2000
2 Feynman R P. Simulating physics with computers. Int J Theor Phys, 1982, 21: 467-488

3 Johanning M, Varon F A, Wunderlich C. Quantum simulations with cold trapped ions. J Phys B: At Mol Opt Phys, 2009, 42: 154009

4 Isenhower L, Urban E, Zhang X L, et al. Demonstration of a neutral atom controlled-NOT quantum gate. Phys Rev Lett, 2010, 104: 010503

5 Wilk T, Gaëtan A, Evellin C, et al. Entanglement of two individual neutral atoms using Rydberg blockade. Phys Rev Lett, 2010, 104: 010502

6 Urban E, Johnson T A, Henage T, et al. Observation of Rydberg blockade between two atoms. Nat Phys, 2009, 5: 110-114

7 Gaëtan A, Miroshnychenko Y, Wilk T, et al. Observation of collective excitation of two individual atoms in the Rydberg blockade regime. Nat Phys, 2009, 5: 115-118

8 Saffman M, Walker T G. Quantum information with Rydberg atoms. Rev Mod Phys, 2010, 82: 2313-2363

9 You L, Yi X X, Su X H. Quantum logic between atoms inside a high-Q optical cavity. Phys Rev A, 2003, 67: 032308

10 Jaksch D, Briegel H-J, Cirac J I, et al. Entanglement of atoms via cold controlled collisions. Phys Rev Lett, 1999, 82: 1975-1978

11 Lee P A, Nagaosa N, Wen X G. Doping a Mott insulator: Physics of high-temperature superconductivity. Rev Mod Phys, 2006, 78: 17-85

12 Yang S, Xu D Z, Song Z, et al. Dimerization-assisted energy transport in light-harvesting complexes. J Chem Phys, 2010, 132: 234501

13 Schlosser N, Reymond G, Protsenko I, et al. Sub-poissonian loading of single atoms in a microscopic dipole trap. Nature, 2001, 411: 1024-1027

14 Schlosser N, Reymond G, Grangier P. Collisional blockade in microscopic optical dipole traps. Phys Rev Lett, 2002, 89: 023005

15 He X D, Xu P, Wang J, et al. Rotating single atoms in a ring lattice generated by a spatial light modulator. Opt Express, 2009, 17: 21014-21021

16 Allen L, Beijersbergen M W, Spreeuw R J C, et al. Orbital angular momentum of light and the transformation of Laguerre-Gaussian laser modes. Phys Rev A, 1992, 45: 8185-8189

17 Kuga T, Torii Y, Shiokawa N, et al. Novel optical trap of atoms with a doughnut beam. Phys Rev Lett, 1997, 78: 4713-4716

18 Franke-Arnold S, Leach J, Padgett M J, et al. Optical ferris wheel for ultracold atoms. Opt Express, 2007, 15: 8619-8625

19 Stutz M, Groblacher S, Jennewein T, et al. How to create and detect $\mathrm{N}$-dimensional entangled photons with an active phase hologram. Appl Phys Lett, 2007, 90: 261114

20 Hanbury-Brown H, Twiss R Q. Correlation between photons in two coherent beams of light. Nature, 1956, 177: 27-29

21 Weber M, Volz J, Saucke K, et al. Analysis of a single-atom dipole trap. Phys Rev A, 2006, 73: 043406

22 He J, Yang B D, Zhang T C, et al. Improvement of the signal-tonoise ratio of laser-induced-fluorescence photon-counting signals of single-atoms magneto-optical trap. J Phys D: Appl Phys, 2011, 44: 135102

23 Bergamini S, Darquie B, Jones M, et al. Holographic generation of microtrap arrays for single atoms by use of a programmable phase modulator. J Opt Soc Am B, 2004, 21: 1889-1894

24 Alt W, Schrader D, Kuhr S, et al. Single atoms in a standing-wave dipole trap. Phys Rev A, 2003, 67: 033403

25 Bali S, O'Hara K M, Gehm M E, et al. Quantum-diffractive background gas collisions in atom-trap heating and loss. Phys Rev A, 1999, 60: R29-R32

26 Savard T A, O'Hara K M, Thomas J E. Laser-noise-induced heating in far-off resonance optical traps. Phys Rev A, 1997, 56: R1095-R1098

27 Landau L D, Lifshitz E M. Mechanics. 3rd ed. London: Butterworth-Heinemann Press, 1998

28 Tuchendler C, Lance A M, Browaeys A, et al. Energy distribution and cooling of a single atom in an optical tweezer. Phys Rev A, 2008, 78: 033425

29 He J, Yang B D, Zhang T C, et al. Extending a release-and-recapture scheme to single atom optical tweezer for effective temperature 
evaluation. Chin Phys B, 2011, 20: 073701

30 Jones M P A, Beugnon J, Gaetan A, et al. Fast quantum state control of a single trapped neutral atom. Phys Rev A, 2007, 75: 040301(R)

31 Beugnon J, Tuchendler $\mathrm{C}$, Marion $\mathrm{H}$, et al. Two-dimensional transport and transfer of a single atomic qubit in optical tweezers. Nat Phys, 2007, 3: 696-699

32 Browaeys A, Häffner H, McKenzie C, et al. Transport of atoms in a quantum conveyor belt. Phys Rev A, 2006, 72: 053605

33 Miroshnychenko Y, Alt W, Dotsenko I, et al. Inserting two atoms into a single optical micropotential. Phys Rev Lett, 2006, 97: 243003

34 He X D, Xu P, Wang J, et al. High efficient loading of two atoms into a microscopic optical trap by dynamically reshaping the trap with a spatial light modulator. Opt Express, 2010, 18: 13586-13592

35 Berry M V. Optical vortices evolving from helicoidal integer and fractional phase steps. J Opt A: Pure Appl Opt, 2004, 6: 259-268

36 Anzolin G, Tamburini F, Bianchini A, et al. Method to measure off-axis displacements based on the analysis of the intensity distribution of a vortex beam. Phys Rev A, 2009, 79: 033845

37 Gallagher A, Pritchard D E. Exoergic collisions of cold $\mathrm{Na}^{*}-\mathrm{Na}$. Phys Rev Lett, 1989, 63: 957-960

38 Weiner J, Bagnato V S, Zilio S, et al. Experiments and theory in cold and ultracold collisions. Rev Mod Phys, 1999, 71: 1-85

39 Wallace C D, Dinneen T P, Tan K Y N, et al. Isotopic difference in trap loss collisions of laser cooled rubidium atoms. Phys Rev Lett, 1992, 69: 897-900

40 Gorges A R, Bingham N S, DeAngelo M K, et al. Light-assisted collisional loss in a ${ }^{85 / 87} \mathrm{Rb}$ ultracold optical trap. Phys Rev A, 2008, 78: 033420

41 Johnson T A, Urban E, Henage T, et al. Rabi oscillations between ground and Rydberg states with dipole-dipole atomic interactions. Phys Rev Lett, 2008, 100: 113003

42 Köhler T, Góral K, Julienne P S. Production of cold molecules via magnetically tunable Feshbach resonances. Rev Mod Phys, 2006, 78: 1311-1361

43 Jones K M, Tiesinga E, Lett P D, et al. Ultracold photoassociation spectroscopy: Long-range molecules and atomic scattering. Rev Mod Phys, 2006, 78: 483-535

44 Ozeri R, Khaykovich L, Davidson N. Long spin relaxation times in a single-beam blue-detuned optical trap. Phys Rev A, 1999, 59: R1750-R1753

45 Puppe T, Schuster I, Grothe A, et al. Trapping and observing single atoms in a blue-detuned intracavity dipole trap. Phys Rev Lett, 2007, 99: 013002

46 Nelson $\mathrm{K}$ D, Li $\mathrm{X}$, Weiss $\mathrm{D} \mathrm{S}$. Imaging single atoms in a three-dimensional array. Nat Phys, 2007, 3: 556-560

47 Isenhower L, Williams W, Dally A, et al. Atom trapping in an inter- ferometrically generated bottle beam trap. Opt Lett, 2009, 34: 1159-1161

48 Davidson N, Lee H J, Adams C S, et al. Long atomic coherence times in an optical dipole trap. Phys Rev Lett, 1995, 74: 1311-1314

49 Saffman M, Walker T G. Analysis of a quantum logic device based on dipole-dipole interactions of optically trapped Rydberg atoms. Phys Rev A, 2005, 72: 022347

$50 \mathrm{Xu} \mathrm{P}, \mathrm{He} \mathrm{X} \mathrm{D}$, Wang J, et al. Trapping a single atom in a blue detuned optical bottle beam trap. Opt Lett, 2010, 35: 2164-2166

51 Yin J P, Gao W J, Wang H F, et al. Generations of dark hollow beams and their applications in laser cooling of atoms and all optical-type Bose-Einstein condensation. Chin Phys, 2002, 11: 1157-1169

52 Chaloupka J L, Meyerhofer D D. Characterization of a tunable, single-beam ponderomotive-optical trap. J Opt Soc Am B, 2000, 17: 713-772

53 Li G, Zhang S, Isenhower L, et al. Crossed vortex bottle beam trap for single-atom qubits. Opt Lett, 2012, 37: 851-853

54 Monroe C, Meekhof D M, King B E, et al. Resolved-sideband Raman cooling of a bound atom to the 3D zero-point energy. Phys Rev Lett, 1995, 75: 4011-4014

55 Hamann S E, Haycock D L, Klose G, et al. Resolved-sideband Raman cooling to the ground state of an optical lattice. Phys Rev Lett, 1998, 80: 4149-4152

56 Roos C F, Leibfried D, Mundt A, et al. Experimental demonstration of ground state laser cooling with electromagnetically induced transparency. Phys Rev Lett, 2000, 85: 5547-5550

57 Leibfried D, Blatt R, Monroe C, et al. Quantum dynamics of single trapped ions. Rev Mod Phys, 2003, 75: 281-324

58 Sortais Y R P, Marion H, Tuchendler C, et al. Diffraction-limited optics for single-atom manipulation. Phys Rev A, 2007, 75: 013406

59 He X D, Yu S, Xu P, et al. Combining red and blue-detuned optical potentials to form a Lamb-Dicke trap for a single neutral atom. Opt Express, 2012, 20: 3711-3724

60 Föster L, Karski M, Choi J M, et al. Microwave control of atomic motion in optical lattices. Phys Rev Lett, 2009, 103: 233001

61 Gallagher T F. Rydberg Atoms. Cambridge: Cambridge University Press, 1994

62 Olmos B, González-Férez R, Lesanovsky I. Collective Rydberg excitations of an atomic gas confined in a ring lattice. Phys Rev A, 2009, 79: 043419

63 Olmos B, González-Férez R, Lesanovsky I. Creating collective many-body states with highly excited atoms. Phys Rev A, 2010, 81: 023604

64 Olmos B, Lesanovsky I. Rydberg rings. Phys Chem Chem Phys, 2011, 13: 4208-4219

Open Access This article is distributed under the terms of the Creative Commons Attribution License which permits any use, distribution, and reproduction in any medium, provided the original author(s) and source are credited. 